\title{
THE MAGNETRON-TYPE VARACTOR FOR FAST CONTROL IN ACCELERATOR RF SYSTEMS
}

\author{
M.I. Kuznetsov, V.V. Paramonov, Yu.V. Senichev \\ Institute for Nuclear Research of the RAS, 117312, Moscow, Russia \\ I.B. Enchevich, R.L. Poirier \\ TRIUMF, Vancouver, B.C., V6T 2A3, Canada
}

\begin{abstract}
The RF test of the magnetron-type varactots have shown [1] the proof of principle of this device. A comparison with the other power rf tunable devices, such as ferrite tuners, shows, that the varactor has a response time a few orders of magnitude shorter. In the experiments this time was estimated to be in the order of $10^{-6}$ sec for operating frequency $\approx 66 \mathrm{M} \mathrm{Hz}$. This feature of the varactor opens new applications of the varactor for a fast modulation (in order of few tens of the working rf period) of the amplitude and the phase of the accelerating voltage in the the rf cavities. Basing on results of numerical simulation, different applications of the device for fast control and improvements in the design to reduce response time are considered.
\end{abstract}

\section{INTRODUCTION}

During the development of rf systems for kaon factories a magnetron type varactor was proposed [4] as a tuner for accelerating cavities in synchrotron rings. The Test\&Development program was carried out as a collaboration between TRIUMF and INR resulting in the proof of principle of this device.

Varactor may be used as the narrow range tuner for accelerating cavities with moderate or high accelerating voltage [1], but main advantage of the magnetron type varactor is its short time of reaction. It stimulates us in consideration of this device as the instrument of fast control in RF systems.

\section{VARACTOR. DESIGN, OPTION OF OPERATION, LIMITATIONS}

A schematic sketch of the varactor is presented in Fig. 1. The varactor is a coaxial system consisting of outer conductor (1), inner conductor (2), cathode (3) and re-ector (4). The ceramic insulator (5) mechanically supports the construction and provides a dc isolation. The magnetic ${ }^{\circledR} \operatorname{eld} B$ is directed along the $z$ axis, the dc control voltage $U_{\mathrm{cc}}$ (with respect to outer conductor) and the rf voltage $U_{\mathrm{rf}}$ are applied between the conductors, providing electric ${ }^{\circledR}$ elds $E_{c C}$ and $E_{\mathrm{rf}}$ in a radial direction. The outer conductor with radius $r_{\mathrm{a}}$ is ${ }^{\circledR x}$ xed at ground potential.

The simple theory of the device is described in [2]. Under conditions above the motion of electrons in interaction space between inner and outer conductors may be subdivided in three parts:

- fast cyclotron rotation with the frequency $\omega_{h}=\left|\frac{e B}{m}\right|$;

- drift of the center of Larmor orbit in azimuth direction with velocity $V \sim\left(E_{c c}+E_{r f}+E_{o}\right) / B$, where $E_{o}$ is the own electric (Beld of the electron cloud;

- motion along $z$ axis.

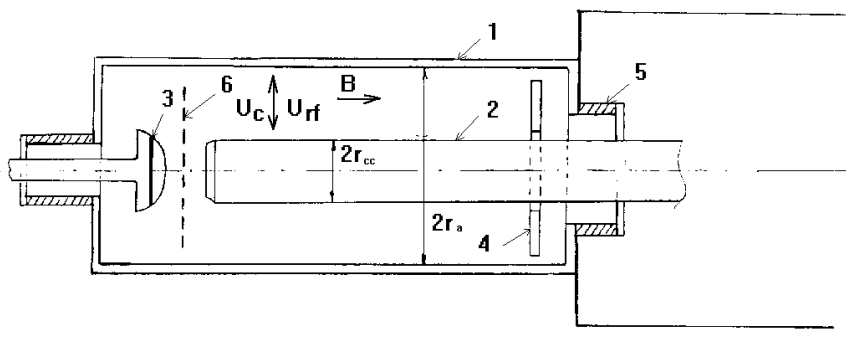

Figure 1. Schematic sketch of the varactor. 1 - outer conductor, 2 - inner conductor, 3 - cathode, 4 - re ector, 5 - insulator, 6 control grid

It may be shown [2], that presence of the electron cloud or electron beam in the interaction space results in an increase of the effective permitivity:

$$
\epsilon=1+\frac{n e^{2}}{m\left(\omega_{h}^{2}-\omega^{2}\right)} \approx 1+\frac{n e^{2}}{m \omega_{h}^{2}}
$$

where $n$ is density of electrons, $\omega=2 \pi f, f$ is the self rf frequency of the system. This effect depends on both the charge density and the position of the electron cloud. Treatment of results obtained in experiments [2] have con®rmed, that the in ${ }^{-} u-$ ence of the charge density is match more stronger, especially if ratio $r_{\mathrm{a}} / r_{\mathrm{cc}}$ is not large.

Several reasons:

a - to have the controllable motion of electrons;

b - to reduce a absorption of the RF energy;

c - to reduce radius of cyclotron rotation, and, hence, radial dimension of the beam - lead to the condition $\omega \ll \omega_{h}$.

Two different options may be realized in the operation of the varactor.

The simplest case is a beam regime, when electron pass varactor one time from the cathode to the re-ector (this case $\mathrm{re}^{-} \mathrm{ec}-$ tor really is an absorber). Only heat loading to the re ector limits the range of tuning. Both estimations and the results of numerical simulation shows for the reasonable heat deposition (less than $1 \mathrm{kWt}$, cooling is necessary) the range $\Delta \epsilon \sim 5 \%$ as achievable. A transit time for electron from the cathode to the absorber may be one order less than RF period. Experiments performed 
in 1992 have shown this option as realistic [3], but re ${ }^{-}$ector must be specially adapted.

Second option of operation - an accumulation of electron cloud, was investigated in more details [2] in 1993. Providing magnetic mirrors near the cathode and the $\mathrm{re}^{-}$ector and prob-

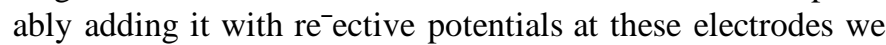
establish a trap for electrons in interaction space. Electron will pass interaction space several times. It leads to suf®cient reduction in the emission current and more uniform distribution of losses in the surface of the device. By providing a good magnetic trap one can get narrow band tuning $(\Delta \epsilon=0 \div 5 \%$ for $U_{\mathrm{cc}}=0 \div-20 \mathrm{kV}$,) at low RF level practically without any drop in $Q$ - factor of the system with very small value of emission current $\left(I_{\mathrm{hc}}=18 \mathrm{~m}\right)$ [2], [1]. Critical points in this option of operation are regions near re $^{-}$ector and near cathode. In these regions density of electrons is match more higher than average density in interaction space (here electrons change sign of axial velocity). If any instabilities take place in the cloud, they arise in these return points. Moreover, main part of an energy extraction from RF Beld takes place under injection and near re-ector. After investigation the results of experiments we have developed the proposals for improvements in injection and the re ${ }^{-}$ector design to reduce RF energy absorption and for damping instabilities. Speci®c time for this mode of operation is life-time of electron in the cloud. From a treatment of experimental data we estimate this time in range $1 \div 10 \mathrm{RF}$ periods, depending on the regime of the varactor (see [2]).

For both options of the varactor operations the response time of the varactor as the controllable device will strongly depend on delay time in a control circuit. Differing from previous versions [4], [3], [1], we consider a now device with constant high DC voltage at the central conductor. Changing of the cloud density will be by modulation of the injection current with low control voltage applied between the cathode and the control grid (Fig.1). All problems, connected with the forming of current pulses in such injection system are good investigated in the development and design of high frequency powerful electron tubes.

The results of numerical simulation have con $®$ rmed previous estimations [1] for reasonable parameters of the device at different levels of the rf voltage and have clarißed reasons of disadvantages.

The reason of nonlinearity in the varactor is the coaxial design. It leads to the nonuniformity of the electric ®elds $E_{\mathrm{CC}}$ and $E_{\mathrm{rf}}$ (the $r^{-1}$ dependence). At low rf voltage level $U_{\text {rf }} \approx 500 \mathrm{~V} \ll$ $U_{c c}$ the displacement of electrons under rf ®eld action is small and the nonlinearity mainly depends on ratio $r_{\mathrm{a}} / r_{\mathrm{cc}}$. At moderate rf voltage level $\left(1 \mathrm{kV} \leq U_{\mathrm{rf}}<U_{\mathrm{cc}}\right)$ additional factor arises. If the density of the cloud is comparable with the Brillouin density $n_{b}=\frac{m \epsilon_{0} \omega_{h}^{2}}{e^{2}}$ (it is needed for $\Delta \epsilon / \epsilon_{\min } \approx 30 \%$ or more), own electric ®eld of the cloud $E_{0}$ is comparable with $E_{\mathrm{CC}}$ and $E_{\mathrm{rf}}$. The displacement of electrons under rf ®eld action is not small and together with high density leads to signi ${ }^{\circledR}$ cant redistribution of electrons in the cloud. This effect grows fast with the increasing of the cloud density $\left(\sim n^{2}\right)$ and very fast with $r_{\mathrm{a}} / r_{\mathrm{cc}}$ increasing. To keep nonlinearity in reasonable limits at moderate rf voltage level we need in restriction $r_{\mathrm{a}} / r_{\mathrm{cc}}<1.5$.

It is known, that all controllable reactive devices are nonlinear. The smallest value of nonlinearity was observed for perpen- dicular biased ferrite-tuned cavities [5]. But in these cavities rf magnetic ®eld is two order less than external control one. For the varactor we are interesting in $U_{\mathrm{rf}} \sim U_{\mathrm{cc}}$ and a nonlinearity don't looks as the especial problem of this device.

For high rf voltage level $U_{\mathrm{rf}} \geq U_{\mathrm{cc}}$ direct numerical simulation of electron motion in the interaction space shows interesting but very complicated picture, especially for high density of the cloud. The dependence of the $E_{o}$ on radius differs from $E_{c c}$ and $E_{r f}$ dependencies - $E_{0}$ grows in absolute value with a radius increasing. The total electric $®$ eld inside the cloud changes with time during rf period $E_{t}=E_{c c}+E_{r f} \sin (\omega t)+E_{o}$. The cloud becomes subdivided with cylindrical surface $r=$ const in two parts, differing in a direction of the azimuthal rotation. As it is known well, such con®guration is unstable. But a boundary between parts moves enough fast in radius due to $E_{r f} \sin (\omega t)$ changing. It provides a very complicated mixture in motion of electron. As it also known well, such effects are very ef®cient in instabilities damping. The ability of stable operation for varactor at high rf voltage level $U_{\mathrm{rf}} \geq U_{\mathrm{cc}}$ have to be proved in experiments.

An application of the varactor looks more comfortable at low frequencies. The scaling relationship for the varactor regimes ( $U_{\mathrm{rf}} \sim U_{\mathrm{cc}} \sim B^{2} \sim \omega_{h}^{2}$ ) is valid. To meet requirement $\omega_{h}^{2} \gg \omega^{2}$, with increasing of operating frequency one have to increase magnetic ${ }^{\circledR}$ eld and control voltage (but will have increase in rf voltage). Another problem is the forming of control pulse at the control grid at high frequency.

\section{POSSIBLE APPLICATIONS OF THE VARACTOR FOR FAST CONTROL}

Experimentally it was shown that the varactor may be used as a controllable reactive device with an rf voltage amplitude at least close to the control voltage value $U_{c c}$. Taking into account all improvements proposed, we consider now the tuning range $\Delta C / C_{\min } \approx 30 \%$ with high $Q$ factor as a reasonable limit at moderate rf voltage level.

With partial connection (through a small dividing capacitor), one may use the varactor for higher values of rf voltage, but with respective reduction in the tuning range.

One can consider the varactor as a part of transmittion line with controllable wave-resistanse or as a capacitor with controllable capacitance. A lot of applications in rf systems are possible, for example phase regulator and so on. Let consider possible applications for fast control of the frequency, and the phase of the accelerating voltage in the the rf cavities.

1. Suppose the varactor is included into a transmission line between an rf ampli®er and a cavity. If one will change the capacitance of the varactor as $C=C_{0}+C_{1} \sin \left(\omega_{1} t\right)$, it will results in amplitude modulation with frequency $\omega_{1}$ for transmittion coef®cient of the line. A rf spectrum for a signal after the varactor will contain frequency components $\omega-\omega_{1}, \omega$ and $\omega+\omega_{1}$. It will be the spectrum of amplitude modulated signal. The cavity will accept this signal if a bandwith $2 \omega_{1}$ is less than a width of a resonant curve of the cavity $\Delta \omega=\omega / Q_{l}$ and rf voltage in the cavity will be modulated in amplitude. Continuing this proposal, one can change the capacitance in more complicated law $C=C_{0}+C_{1} \sin \left(\omega_{1} t\right)+\ldots+C_{m} \sin \left(\omega_{1} m t\right)$. Combin- 
ing $C_{1} \ldots C_{m}$ in a relation de $\AA$ ned one can provide for the cavity $\mathrm{rf}$ spectrum for modulation in frequency (but with a reduction in band, because for modulation in frequency $\omega / Q_{l} \geq 2 m \omega_{1}$. So, using the varactor inserted into transmittion line, one can get in the cavity signal with modulation in amplitude or in frequency, or more complicated signal. The bandwidth of the modulation will be de $\AA$ ned by passband of the cavity. The depth of the modulation depends on the range of capacitance change and can not exceed it. This case seems not a dif®cult problem. The length of the interaction space of the varactor is related with a wavelength at an operating frequency (it is nonef®cient take length $\geq \lambda / 4$ ). Speci®c time for the cloud is also related with operating frequency. For the cavity with reasonable high $Q_{l}$ factor frequency of the modulation is low enough. There will be no dif(B) culties in forming of a pulse on control grid of the varactor.

2. Suppose the varactor is coupled to the cavity by a loop or a probe. One can use the varactor as a delicate fast additional instrument in frequency control system. It may be interesting for accelerating systems with heavy heat loading, for example, linear accelerators in $\mathrm{cw}$ mode of operation. A traditional frequency control by cooling liquid may be enough inertial. If a traditional system will eliminate slow main part of the detuning, the varactor can eliminate a small residual detuning in $Q_{l}$ rf periods. Our calculations, using the coupled circuit method, shows the varactor be able to detune in narrow band tuning range $\Delta \omega / \omega \approx 5.10^{-5}$ enough big cavities, like RFQ or short IH and DTL tanks.

In both cases considered above typical frequency of the modulation is determined by cavity $Q_{l}$ factor. It is more or less evident result, because cavity is mostly inertial chain in the circuit. To provide more fast modulation, one need activly modify parameters of the cavity.

3. Much more interesting results may be obtained when the varactor is directly included in the cavity. Suppose the cavity is good described with lumped parameters, an equivalent capacitance is concentrated near accelerating gap, the varactor is connected in parallel to accelerating gap. If one will change the capacitance of the varactor as $C=C_{0}+C_{1} \sin \left(\omega_{1} t\right)$, it will results in changing of total capacitance of the cavity. Using analogy with LRC-circuits, we have LRC chain with ${ }^{-}$oating parameters. Theoretical analysis of such circuit is very complicated and may be done using methods of a theory of nonlinear oscillations. $\mathrm{Nu}-$ merical simulations shows additional peaks at the resonant curve of such system. The position of peaks at a frequency scale depends on the modulation depth of total capacitance, ratio $\omega_{1} / \omega$ and may be far from the main resonant peak. It means, that the cavity can accept broadband signal and modulation will be faster (really in order of several tens of the working rf period for high $Q_{l}$ cavity). But we consider this conclusion as preliminary. Very careful analysis have to be continued, because such systems (as it is known from theory of oscillations) may exhibit undesirable effects (instability, parasitic oscillations, jumps in phase and amplitude... ).

\section{CONCLUSION}

The magnetron-type varactor now is not the device with speci®ed parameters. It looks now more like principle of operation for new type of controllable reactive RF devices. Our experiments have shown proof of this principle. It is impossible in one paper describe all possible versions and applications. Different particular requirements will lead to different realizations, will need in detailed development and experimental verißcation. Varactor will not replace ferrite-based devices for broad-band applications, but as the instrument for the fast control it has promising perspectives.

\section{References}

[1] I.B. Enchevich et al. RF Test of the Magnetron-Type Varactors, Proc. of 1994 EPAC, v. 3, p. 1980.

[2] I.B. Enchevich et al., RF experiments with the magnetrontype varactors. TRIUMF Design Note TRI-DN-94-16, TRIUMF, Vancouver, 1994.

[3] I.B. Enchevich et al., Experiments with the magnetrontype varactor. TRIUMF Design Note TRI-DN-94-15, TRIUMF, Vancouver, 1994.

[4] V.A. Konovalov, et al., The Perspectives of Application of the Fast-Acting Varactor with Low Losses in High-Current Cyclic and Linear Accelerators. Proc. of the 1992 EPAC Conference, Berlin, v.2, p. 1242, 1990

[5] R.L. Poirier, Perpendicular Biased Ferrite-Tuned Cavities, Proc. of the 1993 IEEE PAC, v.2, p. 753-757, 1994 